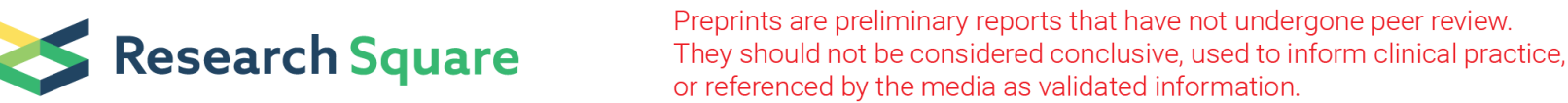

\section{Rice Brittle Culm19 Encoding Cellulose Synthase Subunit CesA4 Causes Dominant Brittle Phenotype But Has No Distinct Influence on Growth and Grain Yield}

\section{Xiaozhi Ma}

Guangdong Academy of Agricultural Sciences

\section{Chunmei Li}

Zhongkai University of Agriculture and Engineering

Rui Huang

Shanxi Agricultural University

\section{Kuan Zhang}

Sichuan Agricultural University - Chengdu Campus

\section{Qian Wang}

Sichuan Agricultural University - Chengdu Campus

Chongyun Fu

Guangdong Academy of Agricultural Sciences

\section{Wuge Liu}

Guangdong Academy of Agricultural Sciences

Changhui Sun

Sichuan Agricultural University - Chengdu Campus

\section{Pingrong Wang}

Sichuan Agricultural University - Chengdu Campus

\section{Feng Wang}

Guangdong Academy of Agricultural Sciences

\section{Xiaojian Deng ( $\nabla$ xjdeng@sicau.edu.cn )}

Sichuan Agricultural University https://orcid.org/0000-0002-0548-0889

\section{Original article}

Keywords: Rice, Dominant mutation, Brittle culm, Cellulose synthase, Secondary cell wall

Posted Date: July 13th, 2021

DOI: https://doi.org/10.21203/rs.3.rs-696804/v1 
License: (c) (i) This work is licensed under a Creative Commons Attribution 4.0 International License. Read Full License

Version of Record: A version of this preprint was published at Rice on November 25th, 2021. See the published version at https://doi.org/10.1186/s12284-021-00536-2. 


\section{Abstract}

Background: Mechanical strength is a crucial agronomic trait in rice (Oryza sativa), and brittle mutants are thought suitable materials to investigate the mechanism of cell wall formation. So far, almost all brittle mutants are recessive, and most of them are defected in multiple morphologies and/or grain yield, limiting their application in hybrid breeding and in rice straw recycling.

Results: We identified a semi-dominant brittle mutant Brittle culm19 (Bc19) isolated from the japonica variety Nipponbare through chemical mutagenesis. The mutant showed the same apparent morphologies and grain yield to the wild type plant except for its weak mechanical strength. Its development of secondary cell wall in sclerenchyma cells was affected, along with reduced contents of cellulose, hemicellulose, lignin and sugars in culms and leaves. Positional cloning suggested that the $B c 19$ gene was allelic to OsCesA4, encoding one of the cellulose synthase A (CesA) catalytic subunits. In this mutant, a C-to-T substitution occurred in the coding sequence of $B C 19$, causing the P507S missense mutation in its encoded product, which was located in the second cytoplasmic region of the OsCesA4 protein. Furthermore, introducing mutant gene Bc19 into the wild-type plant resulted in brittle plants, confirming that the P507S point mutation in OsCesA4 protein was responsible for the semi-dominant brittle phenotype of $B C 19$ mutant. Reverse correlation was revealed between cellulose contents and expression levels of mutant gene $B c 19$ among the homozygous mutant, the hybrid $\mathrm{F}_{1}$ plant, and the $B c 19$ overexpression transgenic plants, implying that gene $B c 19$ might affect cellulose synthesis in a dosagedependent manner.

Conclusions: $B c 19$, a semi-dominant brittle mutant allele of gene OsCesA4, was identified using mapbased cloning approach. The mutated protein of Bc19 possessing the P507S missense mutation behaved in a dosage-dependent semi-dominant manner. Unique brittle effect on phenotype and semidominant genetic quality of gene $B c 19$ indicated its potential application in grain-straw dual-purpose hybrid rice breeding.

\section{Background}

Mechanical strength is one of the most important traits for cereal crops, and is determined by plant cell walls, which constitute the skeletal structures of the plant bodies. Ninety-percent components of plant cell walls are polysaccharides, which exist mainly as cellulose and hemicellulose in primary cell walls (PCW) and secondary cell walls (SCW), and as lignin only in SCW (Darley et al. 2001; Taylor et al. 2000). SCW generally determines the mechanical strength of cell walls, so defects in biosynthesis of cellulose, hemicellulose and/or lignin always result in inferior mechanical index and brittle plant bodies, which in turn make these brittle culm mutants valuable materials for understanding the mechanism of SCW formation.

As a matter of fact, a number of brittle mutants have been studied and some responsible genes have been identified in Arabidopsis, rice and other cereal crops, which to some extent revealed the mechanism 
regulating mechanical strength of the plant body and metabolic pathway of plant cell walls. Initially, physical characteristics of crop stems were first described through three barley brittle mutants (Kokubo et al. 1989; Kokubo et al. 1991). Cellulose, lignin and noncellulose components, maximum bending stresses and mole number of cellulose molecules were determined and compared between mutants and nonbrittle strains, which indicated that weak culms of the three barley mutants were related to decrease in number of cellulose molecules (Kokubo et al. 1991). Later on, three Arabidopsis irregular xylem mutants, irx 1, irx 3 and irx5, were identified (Turner and Somerville 1997), and the relevant corresponding genes were cloned and were confirmed to encode the three basic cellulose synthase A (CesA) catalytic subunits, AtCesA8, AtCesA7 and AtCesA4, respectively (Taylor et al. 1999; Taylor et al. 2000; Taylor et al. 2003). In addition, several genes involved in other different steps of cell wall formation were reported in Arabidopsis. IRX4 encoding a cinnamoyl-CoA reductase (CCR) is essential for lignin biosynthesis; FRAGILE FIBER1 (FRA1) encodes a kinesin-like protein and regulates the oriented deposition of cellulose microfibrils; and mutant of gene FRA2 is attributable to altered fiber cell elongation and expansion (Jones et al. 2001; Zhong et al. 2002; Burk et al. 2001).

\section{Coi}

ncidentally, identification of some brittle mutants in rice caused by Tos 17 also revealed three catalytic subunits essential for cellulose synthesis, OsCesA4, OsCesA7, and OsCesA9, which are homologous to AtCesA8, AtCesA4 and AtCesA7, respectively (Tanaka et al. 2003). Up to now, at least 25 mutants exhibiting brittle leaves and/or culms have been reported in rice, some of which turned out to be different mutant alleles of $C e s A s$, such as $b c 7, b c 11, B c 6$ and $f c 17$ (Yan et al. 2007; Zhang et al. 2009; Kotake et al. 2011; Li et al. 2018). In total, 12 genes responsible for brittle traits are cloned, and they directly or indirectly participate in cellulose biosynthesis and cell wall formation (Kotake et al. 2011; Wu et al. 2012; Vega-Sánchez et al. 2012; Wang et al. 2016b). Brittle Culm1 (BC1) encoding a cobra-like protein regulates cellulose assembly by modulating cellulose crystallite size (Li et al. 2003; Liu et al. 2013). BC3 is suggested to be essential for proper SCW construction, and its encoded protein belonging to a dynamin protein family functions in membrane dynamics (Hirano et al. 2010). A dual-targeting kinesin protein encoded by $B C 12$, which is localized in the nucleus, cytoplasm and mitotic microtubule arrays in dividing cells, is involved in cell-cycle progression and cellulose microfibril deposition (Zhang et al. 2010). Proteins encoded by $B C 10, B C 14$ and $B C 15$ are all localized in the golgi complex, however they function differently in regulating cellulose synthesis (Zhou et al. 2009; Zhang et al. 2016; Zhang et al. 2011; Song et al. 2011; Wu et al. 2012). Characterization of brittle mutants and functional research of the corresponding genes have uncovered some important biochemical processes in cell wall formation and remodeling, while our understanding regarding to cell wall biosynthesis and modification is still limited.

Up to now, almost all brittle mutants are recessive, such as $b c 1, b c 3, b c 11$ and $f c 17$, except one semidominant mutant of OsCesA9 (Li et al. 2003; Hirano et al. 2010; Zhang et al. 2009; Li et al. 2018; Kotake et al. 2011). Meanwhile, except brittle plant bodies, most of them were also aberrant in multiple morphologies, interfering their application in rice breeding. For example, obviously decreased plant height and shorter roots were often seen in these mutants, such as $b c 3, b c 10, b c 11$ and $b c 12$ (Hirano et al. 2010; 
Zhou et al. 2009; Zhang et al. 2009; Zhang et al. 2010). Fertility and/or tillering were even severely affected in some mutants, including NE1031, S1-24, S1-60 and bc10 (Tanaka et al. 2003; Wang et al. 2016a; Wang et al. 2012; Zhou et al. 2009). In the present study, we isolated a semi-dominant brittle mutant Brittle culm19 (BC19) through chemical mutagenesis. Cellulose, hemicellulose and lignin contents were all reduced in culms and leaves of $B c 19$, and its SCW was much thinner than the wild type plant, while neither apparent morphologies nor grain yield was altered in the mutant. Through map-based cloning, we confirmed that the mutant gene Bc19 was allelic to OsCesA4, encoding one of the CesAs, and the resulting P507S point mutation within the second cytoplasmic region of OsCesA4 was responsible for the brittle phenotype. Taken together, we suggest that the semi-dominant mutant gene $B c 19$ could be a potential genetic resource for implement of high grain yield, heavy biomass and their efficient utilization in breeding of grain-straw dual-purpose hybrid rice.

\section{Materials And Methods}

\section{Plant Materials and Growth Conditions}

The Brittle culm19(Bc19) mutant was obtained from the japonica cv. Nipponbare through ethyl methanesulfonate (EMS) mutagenesis. Then Nipponbare, the indica cv. Minghui 63 and G46B were crossed with $B c 19$ to obtain three $F_{1}$ hybrids, respectively. Due to the better polymorphism between Nipponbare and Minghui 63 , the $F_{2}$ mapping population was generated from selfing $F_{1}$ plants of $B c 19$ and Minghui 63. Rice plants were cultivated in the local fields in Wenjiang District (Latitude $30^{\circ} 42^{\prime} \mathrm{N}$, Longitude $103^{\circ} 50^{\prime} \mathrm{E}$, and Altitude $539.3 \mathrm{~m}$ ), Chengdu City, Sichuan, China (Wang et al. 2010).

\section{Measurement of Major Agronomic Traits}

Bc19, Nipponbare, and transgenic plants were cultivated according to randomized complete block design with three replications. Each block contained 40 plants, and the planting density was $16.6 \cdot 25 \mathrm{~cm}$. Field management followed local rice production. Breaking force of flag leaves and the top second internodes were measured two weeks after heading with a digital force testing device (FGJ-1, SHIMPO). The force to break apart a leaf or culm segment was recorded accordingly. Other agronomic traits and yield per plant were investigated after maturation. All of the data were calculated in the software IBM SPSS Statistics 22 , and the statistical significance of differences between $B c 19$ and the wild type plant was conducted using Student's t-test.

\section{Analysis of Cell Wall Components}

Leaves and the top second internodes were collected two weeks after heading, and were dried first at $105^{\circ} \mathrm{C}$ for $1 \mathrm{~h}$, then at $65^{\circ} \mathrm{C}$ for $24 \mathrm{~h}$. Afterwards, the materials were ground into fine powder for measurement. Contents of cellulose, hemicellulose and lignin were measured according to the methods previously described by Van Soest et al (1991). For measurement of cell wall sugars, the powdered materials were soaked in $80 \%$ acetonitrile, and incubated in $65^{\circ} \mathrm{C}$ ultrasonic oscillation water bath for 30 
min. The supernatants were collected through centrifuge, followed by the analysis of monosaccharides with HPLC system (Agilent 1260A) according to the methods previously reported (Zhao et al. 2020).

\section{Electron Microscopy Analysis}

An environmental scanning electron microscope (ESEM, FEl-Q450) was used to observe structure of sclerenchyma and parenchyma tissues in leaves and culms. Flag leaves and the top second internodes were cut into small pieces and then immediately placed on the object stage for observation.

For transmission electron microscope analysis, sections of leaf tissues at seedling stage were treated as previously described (Wang et al. 2010). Generally, these sections were fixed in 3\% (w/v) glutaraldehyde overnight and then in $1 \%$ osmium tetroxide. After that, a gradient of ethanol series were used to dehydrate the samples, followed by washing with the Epon812 medium. Then, the samples were cut into ultra-thin sections and stained with uranyl acetate and Reynolds' lead citrate. Finally, the slices were observed using the transmission electron microscope (H-600IV, Hitachi).

\section{Fine Mapping and Marker Development}

Nine hundred and eighty-three normal plants selected from a $F_{2}$ population up to 5000 plants from the cross between Bc19 and Minghui 63 were used for gene mapping. Genetic linkage analysis was determined using simple sequence repeat (SSR) markers (McCouch et al. 2002) taking genomic DNA as templates. Two DNA pools (normal/brittle), each mixed with 10 individuals, were constructed to screen those SSR markers, which would be further confirmed by the $F_{2}$ segregation population.

Insertion/deletion (InDel) markers were designed using Primer Premier 5.0 software based on genomic DNA sequence polymorphism between japonica and indica from the NCBI website (http://www.ncbi.nlm.nih.gov/BLAST).

\section{Vector Construction and Transgenic Experiment}

The cDNA sequence of the mutant $B c 19$ gene was amplified from the mutant with primers 5 GGGTCTAGAATGATGGAGTCGGGGGTC-3' and 5'-AAAGTCGACTCAGCAGTCGAAGTTGGC-3' (containing a $X$ bal site and a Sal site, respectively), which was inserted into the pMD19-T vector (TaKaRa). After sequencing confirmation, the plasmid was double digested with enzymes $X b a l$ and Sall, subsequently the resulting $B c 19$ fragment was inserted into the binary vector pCAMBIA2300 behind the rice Actin1 promoter. The construct $p C 2300-B c 19$ was introduced into the wild type plant Nipponbare by Agrobacterium tumefaciens-mediated transformation (Kumar et al. 2005). Primers for identifying the transgenic plants were 5'-GAATCCCTCAGCATTGTTC-3' and 5'-TCAAATGTGAGCATAGCC-3', with annealing sites on the rice Actin 1 promoter and CDNA of $B c 19$, respectively. Two homozygous lines (TG1 and TG2) from eleven positive transgenic lines were used for test of cell wall components and for qRTPCR.

\section{Protein Structure Prediction and Sequence Alignment}


Encoded amino acid sequences of CESAs in rice and Arabidopsis were downloaded from GenBank (http://www.ncbi.nlm.nih.gov) through the BLAST program. Protein structures of CesAs were predicted using the TMHMM program (http://www.cbs.dtu.dk/services/TMHMM). Multiple sequence alignments were conducted using DNAMAN software (Lynnon Biosoft).

\section{qRT-PCR Analysis}

Total RNA from leaves and roots of two-week-old seedlings, leaves, culms of plants two weeks after heading, and young panicles at the beginning of heading stage was extracted with a TRIzol reagent (Invitrogen). First-strand cDNA was produced using a Superscript $\triangle$ Reverse Transcription Kit (Invitrogen). Rice Actin 1 gene served as an internal control. Quantitative RT-PCR (qRT-PCR) was performed using a SYBR Premix Ex Taq2 kit (TaKaRa) according to the following program: $95^{\circ} \mathrm{C}$ for $5 \mathrm{~min}$, then 40 cycles of $95^{\circ} \mathrm{C}$ for $10 \mathrm{~s}$ and $58^{\circ} \mathrm{C}$ for $30 \mathrm{~s}$. Specific primers used for qRT-PCR could be seen in Additional file 1: Supplementary Table S3. For each experimental group, qRT-PCR was operated with three technical replicates for each of the three biological replicates.

\section{Results}

\section{Characterization of the Bc19 Mutant}

Bc19 was a brittle mutant obtained from the japonica rice cv. Nipponbare by chemical mutagenesis. Like most of the brittle mutants reported, leaves and internodes of $B c 19$ could be easily broken and the breakpoints were smooth (Fig. 1a, b). The brittle phenotype was milder at seedling stage and became more severe after heading. Not only culms and leaves, the weak mechanical strength went through the whole plant body of $B c 19$, including leaf sheaths, panicle branches, glumes and roots. However, different from most brittle mutants reported, apparent morphology and major agronomic traits were not affected in the $B c 19$ mutant, including plant height, number of productive panicles per plant and spikelets per panicle, seed setting rate, 1000-grain weight, and grain yield (Fig. 1c-i). Strengths for breaking the leaves and culms were measured then, which showed that breaking forces of Bc19 leaves and culms were reduced by approximately $90 \%$ and $70 \%$ of those in the wild-type plant, respectively (Fig. $1 \mathrm{j}, \mathrm{k}$ ).

Decreased mechanical strength in the Bc19 mutant leaves and culms may be a result of irregular cell arrangement, cell wall structure and thickness. Therefore, we examined cross sections of leaves and internodes of the wild-type plant and Bc19 mutant using environmental scanning electron microscope and transmission electron microscope (Fig. 2). Obviously, cell walls of sclerenchyma tissues of the wildtype plant were much thicker than those of Bc19 in both leaf veins and internodes, while cell walls of parenchyma tissues showed no obvious differences between them (Fig. 2a-d). In addition, structures of sclerenchyma tissues, parenchyma tissues, and vascular bundles were not obviously affected in the mutant (Fig. 2a-d). Moreover, SCW of Bc19 was found particularly thinner and the layered structure of SCW was not distinct when compared with the wild-type plant (Fig. 2e-h). These results suggested that the mutation traits of $B c 19$ were very likely associated with decrease in cell wall thickness, especially the SCW thickness in sclerenchyma tissues which provided the basal structural support of the plant body. 


\section{Alterations in Cell Wall Composition}

As alterations in content and percentage of cell wall compositions influence wall structure and mechanical strength of plant bodies, we compared contents of cellulose, hemicellulose, and lignin between the Bc19 mutant and the wild-type plant. As shown in Fig. 3, the amounts of cellulose, hemicellulose and lignin were all reduced in leaves and culms of the Bc19 mutant, when compared with the wild-type plant. Cellulose reductions of $24.1 \%$ in leaves and $28.5 \%$ in culms were observed in $B c 19$, and lignin contents decreased $20.1 \%$ in leaves and $25.9 \%$ in culms compared to those in the wild type (Fig. 3). Relatively slight decrease of hemicellulose content occurred in the mutant, $12.5 \%$ in leaves and $20.9 \%$ in culms (Fig. 3). Subsequently we performed quantitative analysis of monosaccharides in culms of $B c 19$ and wild type plant through HPLC assay. As expected, glucose which make up the cellulose, and xylose which is the main component forming hemicellulose, were all decreased in the $B c 19$ mutant (Table 1). These results indicated that poor mechanical strength of $B c 19$ was probably connected with reduction in components of cell wall, and mutation in gene $B c 19$ affected cell wall biosynthesis through alterations of these major components.

Table 1

Comparison of monosaccharides composition between the wild type (WT) and Bc19.

\begin{tabular}{|c|c|c|c|}
\hline Sugar & WT & $B c 19$ & Compared with WT (\%) \\
\hline Glucose & $9653 \pm 617$ & $7963 \pm 75$ & $-17.5^{\star}$ \\
\hline Fructose & $8568 \pm 455$ & $8135 \pm 259$ & -5.1 \\
\hline Xylose & $321 \pm 38$ & $251 \pm 24$ & $-21.8^{*}$ \\
\hline Arabinose & $8.7 \pm 0.6$ & $8.4 \pm 1.4$ & -3.4 \\
\hline Galactose & $11.3 \pm 0.9$ & $12.2 \pm 1.1$ & 8.0 \\
\hline Rhamnose & $119 \pm 11$ & $123 \pm 3$ & 3.4 \\
\hline Mannose & $48.1 \pm 4.2$ & $61.0 \pm 2.4$ & $26.8^{\star \star}$ \\
\hline \multicolumn{4}{|c|}{$\begin{array}{l}\text { The data are given as means } \pm S E \text { of three independent repeats. Each monosaccharide was } \\
\text { formulated as mg g fresh weight }{ }^{-1} \text {. Single and double asterisks signify statistical differences } \\
\text { compared to the wild type at } P<0.05 \text { and } P<0.01 \text {, respectively. }\end{array}$} \\
\hline
\end{tabular}

\section{Map-based Cloning of the Bc19 Mutant Gene}

The $B c 19$ mutant was crossed with three rice cultivars, including the wild-type Nipponbare, and two indica cultivars, Minghui 63 and G46B. All of the $F_{1}$ plants from the three crossings tended to be brittle. Meanwhile, milder phenotypes of weak mechanical strength and of decrease in breaking force were observed in $\mathrm{F}_{1}$ plants producing from crossing between $B c 19$ and the wild type (Fig. 1a, b, j, k), indicating that $B c 19$ should be a semi-dominant mutant. Consistent with the slight brittle phenotype of the $F_{1}$ 
plants, reduction of cell wall components in $\mathrm{F}_{1}$ were also milder than those in the homozygous mutant of Bc19 (such as $18.9 \%$ and $18.1 \%$ of cellulose decrease in the $F_{1}$ leaves and culms, respectively) (Fig. 3). Therefore, we assumed that the mutant gene acted in a semi-dominant manner rather than completely dominant effect. Furthermore, three quarters of $F_{2}$ plants derived from the three crosses between $B c 19$ and normal cultivars were also brittle plants (Additional file 1: Supplementary Table S1), which indicated that the $B c 19$ mutant phenotype was controlled by a semi-dominant nuclear gene.

In order to fine map the $B c 19$ locus, an $\mathrm{F}_{2}$ population up to 4000 plants from a cross between $B c 19$ and Minghui 63 was generated. Initially, we analyzed 140 normal plants from this $F_{2}$ population with more than 300 SSR markers and located the $B c 19$ gene on the long arm of rice Chromosome 1, with $9.7 \mathrm{cM}$ to RM212 (Fig. 4a). Then several InDel markers on both sides of RM212 were developed based on sequence polymorphism between japonica cv. Nipponbare and indica cv. $93-11$, and the Bc19 gene was mapped between RM212 and InDel marker C1, with 5.1 cM to C1 (Fig. 4a, Additional file 1: Supplementary Table S2). To narrow the Bc19 locus, we designed more InDel markers within this region, analyzed 983 normal plants in total, and finally located Bc19 gene into a 145-kb interval between InDel markers C3 and C4, with the genetic distance of $0.05 \mathrm{cM}$ and $0.10 \mathrm{cM}$, respectively (Fig. 4b, c, Additional file 1: Supplementary Table S2).

According to the gene annotation information provided by the MSU Rice Genome Annotation Release 7 (http://rice.plantbiology.msu.edu), this region totally contains 23 genes (Fig. 4c), of which 8 are transposons. Based on putative functions of the rest 15 genes, gene LOC_Os01g54620 encoding OsCesA4 is most likely the candidate gene, which was previously identified essential for cellulose synthesis (Tanaka et al. 2003). Therefore, we amplified this gene from genomic DNA of Bc19 and wildtype plants, respectively. Sequencing and comparison of them showed one base-pair C-to-T substitution at position 3439 (Fig. 4d). Through reverse transcription (RT)-PCR, the corresponding C-to-T substitution at position 1519 of the gene's CDNA sequence was revealed, turning CCA into TCA and resulting in the 507th amino acid residue, proline, replaced with serine.

Sequence analysis revealed that $B C 19$ gene contained 13 exons and 12 introns with 5743 -bp genomic DNA and 2970-bp cDNA. Multiple alignment indicated that the missense mutation from proline to serine at the 507th residue of $B c 19$ is strictly conserved among CesA family members in rice, Arabidopsis and several other species, which may affect the proper function of OsCesA4 (Fig. 5, Additional file 2: Supplementary Fig. S1).

\section{Functional Identification of $\mathrm{Bc} 19$}

Transgenic experiment was conducted to confirm that mutation in gene LOC_Os01g54620 is responsible for the brittle phenotype of $B c 19$. The plasmid $p C 2300-B c 19$ containing the coding region of the mutant gene $B c 19$ and the rice Actin 1 promoter, was introduced into the wild-type plant. As a result, 13 independent transgenic plants were obtained, of which 11 plants were identified as positive transgenic plants by PCR with a pair of primers extending from the plasmid to the coding sequence of $B c 19$ (Fig. $6 a$, 
showing 2 among 11 lines). As expected, independent transgenic lines carrying the mutant gene $B c 19$ showed very obviously brittle phenotype. Moreover, decrease in cell wall components of transgenic lines TG1 and TG2 were severer than those of mutant $B c 19$ (Fig. 6b, c), probably because of their higher transcript level of mutant gene $B c 19$ guided by the strong promoter of Actin 1 in the recombinant plasmid (Fig. 6d). These results suggested that the brittle phenotype of $B c 19$ mutant was due to the P507S substitution of the OsCesA4 protein, and the mutant $B c 19$ gene blocked synthesis of essential components of cell wall in a dominant way.

\section{qRT-PCR Analysis of BC19 and the Related Genes}

To analyze the function of $B C 19$ gene and in which way the mutant gene impacted its cell wall synthesis, expression patterns of $B C 19$ and other cell wall biosynthesis related genes were assessed in both wildtype plant and the Bc19 mutant by qRT-PCR. We included BC1, OsCesA7, OsCesA9, and OsPAL (Os02g41630) for the analysis. OsCesA4, OsCesA7 and OsCesA9, which encode three catalytic subunits of CesA, are essential for the synthesis of $\beta-1,4$-glucan and are not redundant during formation of SCW (Tanaka et al. 2003; Doblin et al. 2002). BC1 encodes a COBRA-like protein which participates in the formation of SCW, and it was reported that expression of COBRA-like genes closely connected with expression of CesA genes in Arabidopsis (Li et al. 2003; Roudier et al. 2005). OsPAL was reported to regulate lignin synthesis in the phenylpropanoid pathway (Vanholme et al. 2008).

As shown in Fig. 7a, three CesA genes, $B C 1$ and OsPAL were mainly expressed in roots of seedling, culms of plants two weeks after heading and young panicles at the beginning of heading stage, and very low transcript levels of the five genes were detected in leaves at seedling stage and after heading. The only difference was the expression level of OsPAL in young panicles, which was higher than that in culms after heading. Similar expression patterns of $C e s A s$ and $B C 1$ in different tissues suggested that they might be co-expressed in SCW synthesis, which were consistent with the results reported in the study of an OsCesA9 mutant (Kotake et al. 2011). However, they only verified the relationship between OsCesA9 and $B C 1$. Additionally we here confirmed the co-expression pattern between three CesA genes and $B C 1$.

To seek if there was any effect on these related genes' expression caused by the mutated OsCesA4 in the Bc19 mutant, we then examined and compared these genes' expressions between $B c 19$ and the wild type. It turned out that expressions of the five genes were down-regulated in leaves and roots of the mutant, while up-regulated in the $B c 19$ culms and panicles (Fig. 7b). However, changes of transcription levels of these genes were less than two folds in $B c 19$, indicating that the point mutation in $B c 19$ didn't necessarily affect expression of gene $B c 19$ or other related genes. A similar case occurred in the $b c 1$ mutant, where the expression levels of the three CesA genes were not affected by the mutation of gene $B C 1$ (Liu et al. 2013).

In addition, we compared transcript levels of OsCesA4 in the homozygous mutant $B c 19$, the hybrid $\mathrm{F}_{1}$ plant, the wild type and the two particularly brittle transgenic lines TG1 and TG2. As a result, the expression levels in $B c 19$ and $\mathrm{F}_{1}$ plants (the according transcripts probably including both the wild type 
and mutant gene, $B C 19$ and $B C 19$ ) were comparative to those in the wild type. However, sharply raised transcript levels of Bc19 gene were detected in the transgenic lines TG1 and TG2 (Fig. 6d), showing inverse correlation with sharply decreased contents of cellulose, hemicellulose and lignin in them (Fig. 6b, c).

\section{Discussion}

\section{Bc19 Alters Mechanical Strength and Cell Wall Composition but Has No Distinct Influence on Growth and Grain Yield}

Researches on brittle mutants have showed several genes underlying cellulose synthesis and cell wall formation, while mutation of these genes not only affected mechanical strength of plant bodies, but also resulted in other pleiotropic abnormalities in rice. The bc3 mutant showed easily snapped culms, dwarf plant bodies, and short roots (Hirano et al. 2010). Besides brittle culms and leaves, $b c 10$ also exhibited yellowish seedlings, fewer and shorter roots, decreased plant height, and fewer tillers (Zhou et al. 2009). The other brittle mutant, $b c 12$, also showed abnormal growth and morphogenesis, including dwarfism and reduced root length (Zhang et al. 2010). In addition, alterations in cell wall components of these mutants were varied. Briefly, cellulose contents in $b c 3$ and $b c 10$ were decreased but not affected in $b c 12$; lignin content was increased in both $b c 10$ and $b c 12$ (Hirano et al. 2010; Zhou et al. 2009; Zhang et al. 2010).

In case of CesA mutants in rice, different mutation types and sites also resulted in varied phenotypes. NE1031, NC0259, ND8759, and ND2395 were four brittle mutants generated by Tos 17 insertion in OsCesA genes (NE1031 relative to OsCesA4, NC0259 and ND8759 relative to OsCesA7, and ND2395 relative to OsCesA9), and growth and development of them were all aberrant, mainly in plant height, leaf size, culm thickness, and fertility. Moreover, cellulose contents of the four mutants were sharply decreased to $8.9 \%$ $25.5 \%$ of that of the wild type plant (Tanaka et al. 2003). bc7 was another mutant allele of OsCesA4 obtained through $60 \mathrm{Co}-\mathrm{y}$ radiation, resulting in the premature transcription due to the 7-bases deletion in the junction of exon 10 and intron 10 (Yan et al. 2007). Besides weak mechanical strength, plant height of $b c 7$ was slightly shorter than wild type, and cellulose content and cell number of the parenchymatous tissues were all reduced. Other 7 mutants of OsCesA genes were point mutations as reported, including 2 of OsCesA4 (bc11 and fc17), 1 of OsCesA7 (S1-24), and 4 of OsCesA9 (Bc6, S1-60, bc13, and bc-s1) (Zhang et al. 2009; Li et al. 2018; Wang et al. 2016a; Kotake et al. 2011; Wang et al. 2012; Song et al. 2013; Jin et al. 2016). In addition to prominent brittle phenotype, 3 mutants (bc11, S1-24, and S1-60) among them also showed shorter plant bodies, and/or other abnormal morphologies, such as poor fertility, fewer tillers and shorter roots. In most of those mutants, there were reductions in cellulose contents and compensatory increases in hemicellulose and lignin contents, which was different from the brittle mutant in this study (Yan et al. 2007; Wang et al. 2016a; Kotake et al. 2011; Wang et al. 2012; Li et al. 2018). Here, we reported another brittle mutant $B c 19$, in which cellulose, hemicellulose and lignin contents were reduced by $12.5 \%-28.5 \%$, but apparent morphology, growth and grain yield were not affected (Figs. 1, 3). Additionally, different from most recessive brittle mutants such as $f c 17$, we verified 
Bc19 as a new semi-dominant mutant allele of gene OsCesA4, and the mutant gene influenced mainly the SCW synthesis (Figs. 1, 2, 3, 4).

In recent years, constantly raising grain yield attracts breeders' increasing concern of lodging resistance in rice cultivars. Enhancing culm strength can increase the lodging resistance in rice and confrontation capacity against natural disasters such as strong wind and rain ( $\mathrm{Li}$ et al. 2014a). On the other hand, reduced proportion of components in cell walls could make rice straw easily degraded after harvest, improving its utilization efficiency either as animal feed or biofuels (Wang et al. 2005; Johnson et al. 2006). In addition, engineering improvement of rice straw as biofuel resources will be a sustainable solution to solve environmental problems owing to straw burning (Himmel et al. 2007; Ragauskas et al. 2014). In this study, cellulose content was reduced while growth and yield were not affected in $B c 19$ mutant, thus the mutant gene could be a potential genetic resource for rice straw recycling, which could maintain grain yield as well (Figs. 1,3). What's more, the specific semi-dominant characteristic of brittle gene $B c 19$ makes it more convenient to implement of high grain yield, heavy biomass and their efficient utilization in breeding of grain-straw dual-purpose hybrid rice (Peng et al. 2010; Li et al. 2019).

\section{The Point Mutation in Bc19 Could Partially Abolish OsCesA4 Function}

OsCesA4, together with OsCesA7 and OsCesA9, encoding CesAs, are indispensable and irredundant during biosynthesis of cell wall in rice (Tanaka et al. 2003). The three OsCesA subunits share strong similarity in amino acid sequences, and they possess similar function domains including a Zinc Finger domain, a D,D,D,QXXRW motif, a Plant-conserved Region (P-CR), a Class-specific Region (C-SR) and totally 8 transmembrane domains (TMDs) (Somerville 2006; Li et al. 2014b) (Fig. 8). Recently it was visually identified that the PttCesA8 subunit in poplar (Populus tremula $\times$ tremuloides) possessed 7 TMDs, but none of such discoveries was found in rice or other species (Purushotham et al. 2020). Nevertheless, the functional cellulose synthase complex (CSC) has been identified as a hexamer, the socalled rosette structure which is anchored to the plasma membrane (Atanassov et al. 2009). Through interactions induced by the Zinc Finger domain with each other, single CesA subunit first forms homodimers and then six homodimers are polymerized into the rosette structure (Kurek et al. 2002; Timmers et al. 2009; Hill et al. 2014; Nixon et al. 2016). Although formation of the rosette structure relies on what motif of CesA subunits remains unclear, it is predicted that the D,D,D,QXXRW motif as well as the P-CR in the second cytoplasmic region between TMD2 and TMD3 are essential for the catalytic activity during cellulose synthesis (Taylor et al. 2000; Doblin et al. 2002; Arioli et al. 1998; Rushton et al. 2017). In another word, a functional CSC must possess three elements, correct Zinc Finger motif to form the rosette, correct second cytoplasmic region for the catalytic activity, and correct TMDs for locating to the plasma membrane.

NE1031, bc11 and Bc19 are allelic mutants of OsCesA4 with different mutation sites, while they exhibit different morphological abnormalities. NE1031 was found the Tos17 insertion in the sixth exon, which resulted in the premature transcription of OsCesA4 (Tanaka et al. 2003). The point mutation in $b c 11$ was located in the end of TMD5, resulting in decreased abundance of OsCESA4 in the plasma membrane 
(Zhang et al. 2009). Despite weak mechanical strength, these two mutants also displayed dwarfed plant bodies; and NE1031 even exhibited small leaves, thinner culms and low fertility. Moreover, cellulose contents were all sharply reduced in NE1031 and $b c 11$, by $79.6 \%$ and $57 \%$, respectively. In this study, the P507S mutation within the second cytoplasmic region of OsCesA4 only influenced the mechanical strength of $B c 19$, accompanied by $28.5 \%$ reduction in cellulose content and thinner SCW (Figs. 1, 2, 3). Meanwhile, transcript levels of $B c 19$ were not apparently affected in the mutant (Figs. 6d, 7b). Considering normal Zinc Finger domain and TMDs of OsCesA4, the rosette probably could still be secreted and trafficked to the plasma membrane in Bc19. Evidence was that in the irx 1 (AtCesA8) mutant in Arabidopsis, antibodies specifically combining to IRX3 (AtCesA7) and IRX5 (AtCesA7) could still coprecipitate IRX1, suggesting that the point mutation in the third Asp residue of the D,D,D,QXXRW motif in mutant irx 1 didn't affect interactions of the three kinds of CesA subunits (Taylor et al. 2000; Taylor et al. 2003). Therefore, different from complete loss of function of OsCesA4 in NE1031 due to its premature transcription termination, catalytic efficiency of the mutated OsCesA4 in Bc19 might just be reduced to some extent, instead of thoroughly abolished. After all, cellulose content in $B c 19$ was not sharply reduced as it was in NE1031 (Fig. 3) (Tanaka et al. 2003).

\section{Possible Explanation for the Dominant Negative Effect of the Point Mutation in Bc19}

Although more than 20 brittle mutants have been reported in rice, almost all of them were recessive, and the only exception was Bc6, a semi-dominant mutant allele of OsCesA9 in rice (Kotake et al. 2011). In this study, we characterized another semi-dominant brittle mutant Bc19, which was allelic to OsCesA4. Till now, 8 single-base substitution mutant alleles of CesA proteins have been located on different functional domains of them (Fig. 8). S1-24, mutated on the Zinc Finger domain of protein OsCesA7, was presumed to affect interactions of OsCesA7 with other CesA subunits, and the mutant phenotype was recessive (Wang et al. 2016a). Similar events occurred in mutant $b c 13$ and $b c-s 1$, of which the missense mutations were mapped closely before and behind the Zinc Finger domain, respectively. Recessive mutants $b c 11$ and S1-60, possessing point mutations at the beginning and the end of TMD5, respectively, were speculated to impede enrichment of the corresponding CesA subunits (OsCesA4 and OsCesA9) on the plasma membrane (Fig. 8) (Zhang et al. 2009; Wang et al. 2012). Furthermore, over-expression of the mutated cDNA of $b c 11$ in the $b c 11$ mutant background unexpectedly rescued the brittle phenotype, suggesting that excessive amount of the mutant version of OsCesA4 could make up the abundance of OsCesA4 in the plasma membrane, which implied that this point mutation did not change its normal catalytic activity, and might only affect secretion of the complex from the endomembrane to the plasma membrane (Zhang et al. 2009). As for the two semi-dominant mutants, Bc6 reported before and Bc19 in this study, the former one carrying a R588G mutation in OsCesA9 and the latter one with a P507S mutation in OsCesA4, both of them mutated in the second cytoplasmic domain between TMD2 and TMD3 (Kotake et al. 2011). Multiple alignment of CesA subunits from rice and other species showed that the interval between the P507 of OsCesA4 and the R588 of OsCesA9 was only one amino acid (Fig. 5). It's worth noting that the P507 residue is highly conserved among CesA family members, and it exactly corresponds to P557 of AtCesA7, which was changed to a threonine residue in the semi-dominant mutant fra5 in Arabidopsis (Figs. 5, 8) (Zhong et al. 2003). 
Controlling mechanism of whether a particular mutated CesA subunit acts in a dominant or recessive way might be associated with different mutation sites in different function domains of them. In the study of fra5, it was hypothesized that the second cytoplasmic domain might participate in interactions of CesAs with other components in the rosette complex, and the missense mutation in fra5 perturbed this kind of interaction and then affected the normal function of CSC, which resulted in the dominant negative effect (Zhong et al. 2003). However, Kotake et al. (2011) speculated that the P-CR of the second cytoplasmic domain might be involved in interactions between CesA subunits during the formation of higher order CesA oligomerization, so that the mutation in $B c 6$ might interfere the formation of functional CSC. In this study, overexpression of Bc19 in the wild type caused more severe brittle phenotype than the $B c 19$ mutant, while the mutant phenotype of $F_{1}$ plants was milder than $B c 19$ (Figs. $6 \mathrm{~b}-\mathrm{d}, 3,1 \mathrm{a}, \mathrm{b}, \mathrm{j}, \mathrm{k}$ ). It seems that the severity of the mutant phenotype is tightly related to the level of $B c 19$ expression, implying that despite the dominant-negative effect, $B c 19$ might also act in a dosage way. Considering these characteristics of mutant gene $B c 19$, we raise the possibility here that in the hybrid $F_{1}$ plants, the encoded product of the mutant gene Bc19 can randomly bind to the CSC and can be secreted to the plasma membrane together with the normal CSCs, but the abnormal CSCs cannot catalyze cellulose synthesis as efficiently as the normal CSC, resulting in milder cellulose deficiency compared to the homozygous mutant. Similarly, in the BC19 overexpression plants, even more abnormal CSCs carrying the mutated OsCesA4 subunit gather onto the plasma membrane, thus severely impacting efficiency of cellulose synthesis, which fits the dominant-negative effect of a dose-dependent fashion. In cases of hybrid plants of point mutations in Zinc Finger domain or TMDs, abnormal subunits encoded by mutated genes either cannot form the CSC or cannot be secreted to the plasma membrane, thus CSCs transferred onto the plasma membrane are all functional CSCs compromising of normal subunits encoded by the correct genes, which can efficiently catalyze cellulose synthesis, and that's why these mutated genes act in recessive ways. Intensive exploration on action mechanism of P-CR of CesA subunits and on activities between components within the CSC complex will help to uncover how these P-CRs participate in cellulose biosynthesis.

\section{Conclusions}

Bc19, a semi-dominant brittle mutant allele of gene OsCesA4, was identified using map-based cloning approach. Breaking force and cellulose content of the $B c 19$ mutant were decreased, while its apparent morphology, growth and grain yield were not affected. The product encoded by $B c 19$ possessed a P507S missense mutation located in the second cytoplasmic region, causing the dominant-negative effect of gene $B c 19$. The relationship between expression levels of $B c 19$ and severity of brittle phenotype further revealed that gene $B c 19$ might affect cellulose synthesis in a dosage-dependent manner. Moreover, we presumed that the $B c 19$ gene could be a potential genetic resource in breeding of grain-straw dualpurpose hybrid rice.

\section{Abbreviations}


CesA: cellulose synthase A; PCW: primary cell walls; SCW: secondary cell walls; P-CR: Plant-conserved Region; C-SR: Class-specific Region; TMD: transmembrane domains; CSC: cellulose synthase complex

\section{Declarations}

\section{Acknowledgments}

We are grateful to Prof. Chengcai Chu for kindly providing pCAMBIA2300 vector (Institute of Genetics and Developmental Biology, Chinese Academy of Sciences, Beijing, China).

\section{Authors' contributions}

$\mathrm{XM}$ and $\mathrm{CL}$ performed the experiments, and $\mathrm{XM}$ wrote the manuscript. $\mathrm{RH}, \mathrm{KZ}, \mathrm{QW}$ and $\mathrm{CF}$ conducted the field experiments. WL, CS and PW helped managing the main data analysis. XD and FW designed the study and critically revised the article.

\section{Funding}

This study was supported by the National Natural Science Foundation of China (31901531 and 31971869), the Natural Science Foundation of Guangdong Province (2017A030310290, 2019A1515110867 and 2020A1515011052), Guangdong Province Scientific and Technological Plan Project (2018B020202004 and 2020B1212060047), and the Agricultural Competitive Industry Discipline Team Building Project of Guangdong Academy of Agricultural Sciences.

\section{Availability of Data and Materials}

All data generated or analyzed during this study are included in this article (and its supplementary information files).

\section{Ethics Approval and Consent to Participate}

Not applicable.

\section{Consent for Publication}

Not applicable.

\section{Competing Interests}

The authors declare no potential competing interests.

\section{Author details}

${ }^{1}$ State Key Laboratory of Crop Gene Exploration and Utilization in Southwest China, Rice Research Institute, Sichuan Agricultural University, Chengdu 611130, China. ${ }^{2}$ Guangdong Provincial Key Laboratory 
of New Technology in Rice Breeding, Rice Research Institute, Guangdong Academy of Agricultural Sciences, Guangzhou 510642, China. ${ }^{3}$ Guangzhou Key Laboratory for Research and Development of Crop Germplasm Resources, College of Agriculture and Biology, Zhongkai University of Agriculture and Engineering, Guangzhou 510225, China. ${ }^{4}$ Center for Agricultural Genetic Resources Research, Shanxi Agricultural University, Taiyuan 030031, China.

\section{References}

1. Arioli T, Peng LC, Betzner AS, Burn J, Wittke W, Herth W, Camilleri C, Höfte H, Plazinski J, Birch R, Cork A, Glover J, Redmond J, Williamson RE (1998) Molecular analysis of cellulose biosynthesis in Arabidopsis. Science 279(5351):717-720

2. Atanassov II, Pittman JK, Turner SR (2009) Elucidating the mechanism of assembly and subunit interaction of the cellulose synthase complex of Arabidopsis secondary cell walls. J Biol Chem 284(6):3833-3841

3. Burk DH, Liu B, Zhong RQ, Morrison WH, Ye ZH (2001) A katanin-like protein regulates normal cell wall biosynthesis and cell elongation. Plant Cell 13(4):807-827

4. Darley CP, Forrester AM, McQueen-Mason SJ (2001) The molecular basis of plant cell wall extension. Plant Mol Biol 47(1-2):179-195

5. Doblin MS, Kurek I, Jacob-Wilk D, Delmer DP (2002) Cellulose biosynthesis in plants: from genes to rosettes. Plant Cell Physiol 43(12):1407-1420

6. Hill JL Jr, Hammudi MB, Tien M (2014) The Arabidopsis cellulose synthase complex: a proposed hexamer of CESA trimers in an equimolar stoichiometry. Plant Cell 26(12):4834-4842

7. Himmel ME, Ding SY, Johnson DK, Adney WS, Nimlos MR, Brady JW, Foust TD (2007) Biomass recalcitrance: engineering plants and enzymes for biofuels production. Science 315(5813):804-807

8. Hirano K, Kotake T, Kamihara K, Kamihara K, Tsuna K, Aohara T, Kaneko Y, Takatsuji H, Tsumuraya Y, Kawasaki S (2010) Rice BRITTLE CULM 3 (BC3) encodes a classical dynamin OsDRP2B essential for proper secondary cell wall synthesis. Planta 232(1):95-108

9. Jin ZM, Ping BZ, Shen HJ, Du HQ, Li RQ, Zhu L, Zhang DB, Yuan Z (2016) Characterization and gene mapping of a brittle culm mutant $b c-s 1$ in rice. Chin Bull Bot 51(2):167-174. (In Chinese with English abstract)

10. Johnson SE, Angeles OR, Brar DS, Buresh RJ (2006) Faster anaerobic decomposition of a brittle straw rice mutant: implications for residue management. Soil Biol Biochem 38(7):1880-1892

11. Jones L, Ennos AR, Turner SR (2001) Cloning and characterization of irregular xylem4 (irx4): a severely lignin-deficient mutant of Arabidopsis. Plant J 26(2):205-216

12. Kokubo A, Kuraishi S, Sakurai N (1989) Culm strength of barley: correlation among maximum bending stress, cell wall dimensions, and cellulose content. Plant Physiol 91(3):876-882

13. Kokubo A, Sakurai N, Kuraishi S, Takeda K (1991) Culm brittleness of barley (Hordeum vulgare L.) mutants is caused by smaller number of cellulose molecules in cell wall. Plant Physiol 97(2):509- 


\section{4}

14. Kotake T, Aohara T, Hirano K, Sato A, Kaneko Y, Tsumuraya Y, Takatsuji H, Kawasaki S (2011) Rice Brittle culm 6 encodes a dominant-negative form of CesA protein that perturbs cellulose synthesis in secondary cell walls. J Exp Bot 62(6):2053-2062

15. Kumar KK, Maruthasalam S, Loganathan M, Sudhakar D, Balasubramanian P (2005) An improved Agrobacterium-mediated transformation protocol for recalcitrant elite indica rice cultivars. Plant Mol Biol Rep 23(1):67-73

16. Kurek I, Kawagoe Y, Jacob-Wilk D, Doblin M, Delmer D (2002) Dimerization of cotton fiber cellulose synthase catalytic subunits occurs via oxidation of the zinc-binding domains. Proc Natl Acad Sci U S A 99(17):11109-11114

17. Li FC, Liu ST, Xu H, Xu Q (2018) A novel FC17/CESA4 mutation causes increased biomass saccharification and lodging resistance by remodeling cell wall in rice. Biotechnol Biofuels 11(1):298

18. Li FC, Zhang ML, Guo K, Hu Z, Zhang R, Feng YQ, Yi XY, Zou WH, Wang LQ, Wu CY, Tian JS, Lu TG, Xie GS, Peng LC (2014a) High-level hemicellulosic arabinose predominately affects lignocellulose crystallinity for genetically enhancing both plant lodging resistance and biomass enzymatic digestibility in rice mutants. Plant Biotechnol 13(4):514-525

19. Li J, Fang ZW, Wang BX, Yang B, Chi M, Lu BG, Liu Y, Chen TM, Liu JB, Xing YG, Xu B, Sun ZG, Xu DY (2019) Comparative analysis of main characteristics of new brittle culm Japonica rice lines. China Rice 25(6):100-102. (In Chinese with English abstract)

20. Li SD, Bashline L, Lei L, Gu Y (2014b) Cellulose synthesis and its regulation. The Arabidopsis Book 12:e0169

21. Li YH, Qian Q, Zhou YH, Yan MX, Sun L, Zhang M, Fu ZM, Wang YH, Han B, Pang XM, Chen MS, Li JY (2003) BRITTLE CULM1, which encodes a COBRA-like protein, affects the mechanical properties of rice plants. Plant Cell 15(9):2020-2031

22. Liu LF, Shang-Guan KK, Zhang BC, Liu XL, Yan MX, Zhang LJ, Shi YY, Zhang M, Qian Q, Li JY, Zhou YH (2013) Brittle Culm1, a COBRA-like protein, functions in cellulose assembly through binding cellulose microfibrils. PLoS Genet 9(8):e1003704

23. McCouch SR, Teytelman L, Xu YB, Lobos KB, Clare K, Walton M, Fu BY, Maghirang R, Li ZK, Xing YZ, Zhang QF, Kono I, Yano M, Fjellstrom R, Declerck G, Schneider D, Cartinhour S, Ware D, Stein L (2002) Development and mapping of 2240 new SSR markers for rice (Oryza sativa L.). DNA Res 9(6):199207

24. Nixon BT, Mansouri K, Singh A, Du J, Davis JK, Lee JG, Slabaugh E, Vandavasi VG, Neill HO, Roberts EM, Roberts AW, Yingling YG, Haigler CH (2016) Comparative structural and computational analysis supports eighteen cellulose synthases in the plant cellulose synthesis complex. Sci Rep 6:28696

25. Peng YC, Gan CH, Wang HT, Xu ZJ (2010) Breeding of dominant brittle culm CMS line Zhongcui A in rice. Hybrid Rice 25(4):9-13. (In Chinese with English abstract)

26. Purushotham P, Ho R, Zimmer J (2020) Architecture of a catalytically active homotrimeric plant cellulose synthase complex. Science 369(6507):1089-1094 
27. Ragauskas AJ, Beckham GT, Biddy MJ, Chandra R, Chen F, Davis MF, Davison BH, Dixon RA, Gilna P, Keller M, Langan P, Naskar AK, Saddler JN, Tschaplinski TJ, Tuskan GA, Wyman CE (2014) Lignin valorization: improving lignin processing in the biorefinery. Science 344(6185):1246843

28. Roudier F, Fernandez AG, Fujita M, Himmelspach R, Borner GHH, Schindelman G, Song S, Baskin TI, Dupree P, Wasteneys GO (2005) COBRA, an Arabidopsis extracellular glycosyl-phosphatidyl inositolanchored protein, specifically controls highly anisotropic expansion through its involvement in cellulose microfibril orientation. Plant Cell 17(6):1749-1763

29. Rushton PS, Olek AT, Makowski L, Badger J, Steussy CN, Carpita NC, Stauffacher CV (2017) Rice cellulose synthaseA8 plant-conserved region is a coiled-coil at the catalytic core entrance. Plant Physiol 173(1):482-494

30. Somerville C (2006) Cellulose synthesis in higher plants. Annu Rev Cell Dev Biol 22:53-78

31. Song XQ, Liu LF, Jiang YJ, Zhang BC, Gao YP, Liu XL, Lin QS, Ling HQ, Zhou YH (2013) Disruption of secondary wall cellulose biosynthesis alters cadmium translocation and tolerance in rice plants. Mol Plant 6(3):768-780

32. Song XQ, Zhang BC, Zhou YH (2011) Golgi-localized UDP-glucose transporter is required for cell wall integrity in rice. Plant Signal Behav 6(8):1097-1100

33. Tanaka K, Murata K, Yamazaki M, Onosato K, Miyao A, Hirochika H (2003) Three distinct rice cellulose synthase catalytic subunit genes required for cellulose synthesis in the secondary wall. Plant Physiol 133(1):73-83

34. Turner SR, Somerville CR (1997) Collapsed xylem phenotype of Arabidopsis identifies mutants deficient in cellulose deposition in the secondary cell wall. Plant Cell 9(5):689-701

35. Taylor NG, Howells RM, Huttly AK, Vickers K, Turner SR (2003) Interactions among three distinct CesA proteins essential for cellulose synthesis. Proc Natl Acad Sci U S A 100(3):1450-1455

36. Taylor NG, Laurie S, Turner SR (2000) Multiple cellulose synthase catalytic subunits are required for cellulose synthesis in Arabidopsis. Plant Cell 12(12):2529-2539

37. Taylor NG, Scheible W, Cutler S, Somerville CR, Turner SR (1999) The irregular xylem3 locus of Arabidopsis encodes a cellulose synthase required for secondary cell wall synthesis. Plant Cell 11(5):769-779

38. Timmers J, Vernhettes S, Desprez T, Vincken J-P, Visser RGF, Trindade LM (2009) Interactions between membrane-bound cellulose synthases involved in the synthesis of the secondary cell wall. FEBS Lett 583(6):978-982

39. Van Soest PJ, Robertson JB, Lewis BA (1991) Methods for dietary fiber, neutral detergent fiber, and nonstarch polysaccharides in relation to animal nutrition. J Dairy Sci 74(10):3583-3598

40. Vanholme R, Morreel K, Ralph J, Boerjan W (2008) Lignin engineering. Curr Opin Plant Biol 11(3):278-285

41. Vega-Sánchez ME, Verhertbruggen $Y$, Christensen U, Chen XW, Sharma V, Varanasi P, Jobling SA, Talbot M, White RG, Joo M, Singh S, Auer M, Scheller HV, Ronald PC (2012) Loss of cellulose 
synthase-like F6 function affects mixed-linkage glucan deposition, cell wall mechanical properties, and defense responses in vegetative tissues of rice. Plant Physiol 159(1):56-69

42. Wang DF, Qin YL, Fang JJ, Yuan SJ, Peng LX, Zhao JF, Li XY (2016a) A missense mutation in the zinc finger domain of OsCESA7 deleteriously affects cellulose biosynthesis and plant growth in rice. PLoS One 11(4):e0153993

43. Wang DF, Yuan SJ, Yin L, Zhao JF, Guo BT, Lan JH, Li XY (2012) A missense mutation in the transmembrane domain of CESA9 affects cell wall biosynthesis and plant growth in rice. Plant Sci 196:117-124

44. Wang HF, Zhu JL, Liu JX, Wu YM, Qian Q, Teng S (2005) Growth performance, nutrient digestibility and carcass quality of growing-finishing pigs fed different levelsp; of whole crop rice Brittle Culm-1. Acta Veterinaria et Zootechnica Sinica 36(11):1139-1144. (In Chinese with English abstract)

45. Wang PR, Gao JX, Wan CM, Zhang FT, Xu ZJ, Huang XQ, Sun XQ, Deng XJ (2010) Divinyl chlorophyll(ide) a can be converted to monovinyl chlorophyll(ide) a by a divinyl reductase in rice. Plant Physiol 153(3):994-1003

46. Wang XL, Cheng ZJ, Zhao ZC, Gan L, Qin RZ, Zhou KN, Ma WW, Zhang BC, Wang JL, Zhai HQ, Wan JM (2016b) BRITTLE SHEATH1 encoding OsCYP96B4 is involved in secondary cell wall formation in rice. Plant Cell Rep 35(4):745-755

47. Wu B, Zhang BC, Dai Y, Zhang L, Shang-Guan KK, Peng YG, Zhou YH, Zhu Z (2012) Brittle Culm15 encodes a membrane-associated chitinase-like protein required for cellulose biosynthesis in rice. Plant Physiol 159(4):1440-1452

48. Yan CJ, Yan S, Zeng XH, Zhang ZQ, Gu MH (2007) Fine mapping and isolation of $B c 7(t)$, allelic to OsCesA4. J Genet Genomics 34(11):1019-1027

49. Zhang BC, Deng LW, Qian Q, Xiong GY, Zeng DL, Li R, Guo LB, Li JY, Zhou YH (2009) A missense mutation in the transmembrane domain of CESA4 affects protein abundance in the plasma membrane and results in abnormal cell wall biosynthesis in rice. Plant Mol Biol 71(4-5):509-524

50. Zhang BC, Liu XL, Qian Q, Liu LF, Dong GJ, Xiong GY, Zeng DL, Zhou YH (2011) Golgi nucleotide sugar transporter modulates cell wall biosynthesis and plant growth in rice. Proc Natl Acad Sci U S A 108(12):5110-5115

51. Zhang M, Zhang BC, Qian Q, Yu YC, Li R, Zhang JW, Liu XL, Zeng DL, Li JY, Zhou YH (2010) Brittle Culm 12, a dual-targeting kinesin-4 protein, controls cell-cycle progression and wall properties in rice. Plant J 63(2):312-328

52. Zhang ML, Wei F, Guo K, Hu Z, Li YY, Xie GS, Wang YT, Cai XW, Peng LC, Wang LQ (2016) A novel FC116/BC10 mutation distinctively causes alteration in the expression of the genes for cell wall polymer synthesis in rice. Front Plant Sci 7:1366

53. Zhao YT, Yue ZC, Zhong XM, Lei JL, Tao P, Li BY (2020) Distribution of primary and secondary metabolites among the leaf layers of headed cabbage (Brassica oleracea var. capitata). Food Chem $312: 126028$ 
54. Zhong RQ, Burk DH, Morrison WH III, Ye ZH (2002) A kinesin-like protein is essential for oriented deposition of cellulose microfibrils and cell wall strength. Plant Cell 14(12):3101-3117

55. Zhong RQ, Morrison WH III, Freshour GD, Hahn MG, Ye ZH (2003) Expression of a mutant form of cellulose synthase AtCesA7 causes dominant negative effect on cellulose biosynthesis. Plant Physiol 132(2):786-795

56. Zhou YH, Li SB, Qian Q, Zeng DL, Zhang M, Guo LB, Liu XL, Zhang BC, Deng LW, Liu XF, Luo GZ, Wang XJ, Li JY (2009) BC10, a DUF266-containing and Golgi-located type II membrane protein, is required for cell-wall biosynthesis in rice (Oryza sativa L.). Plant J 57(3):446-462

\section{Figures}



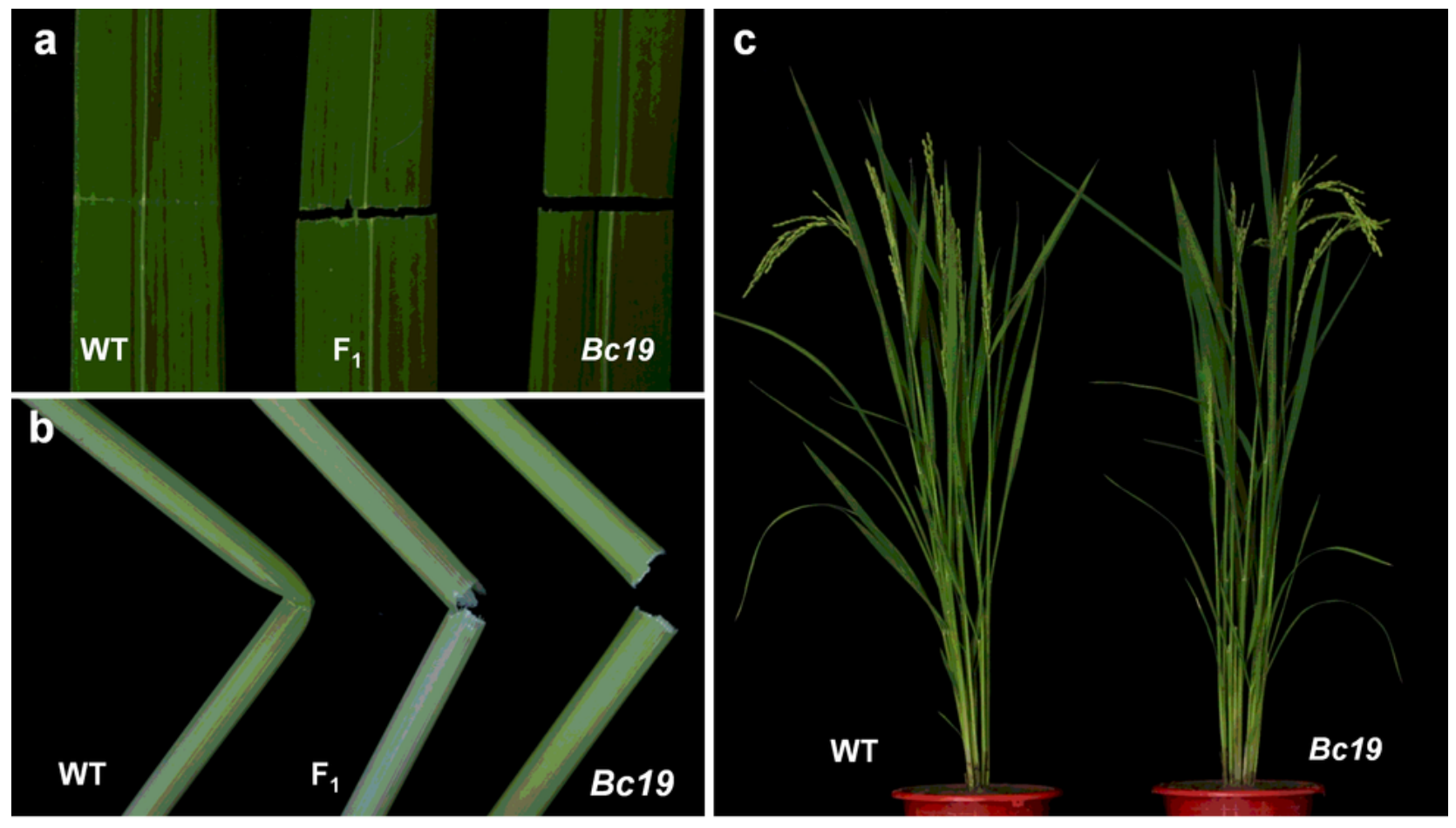

h
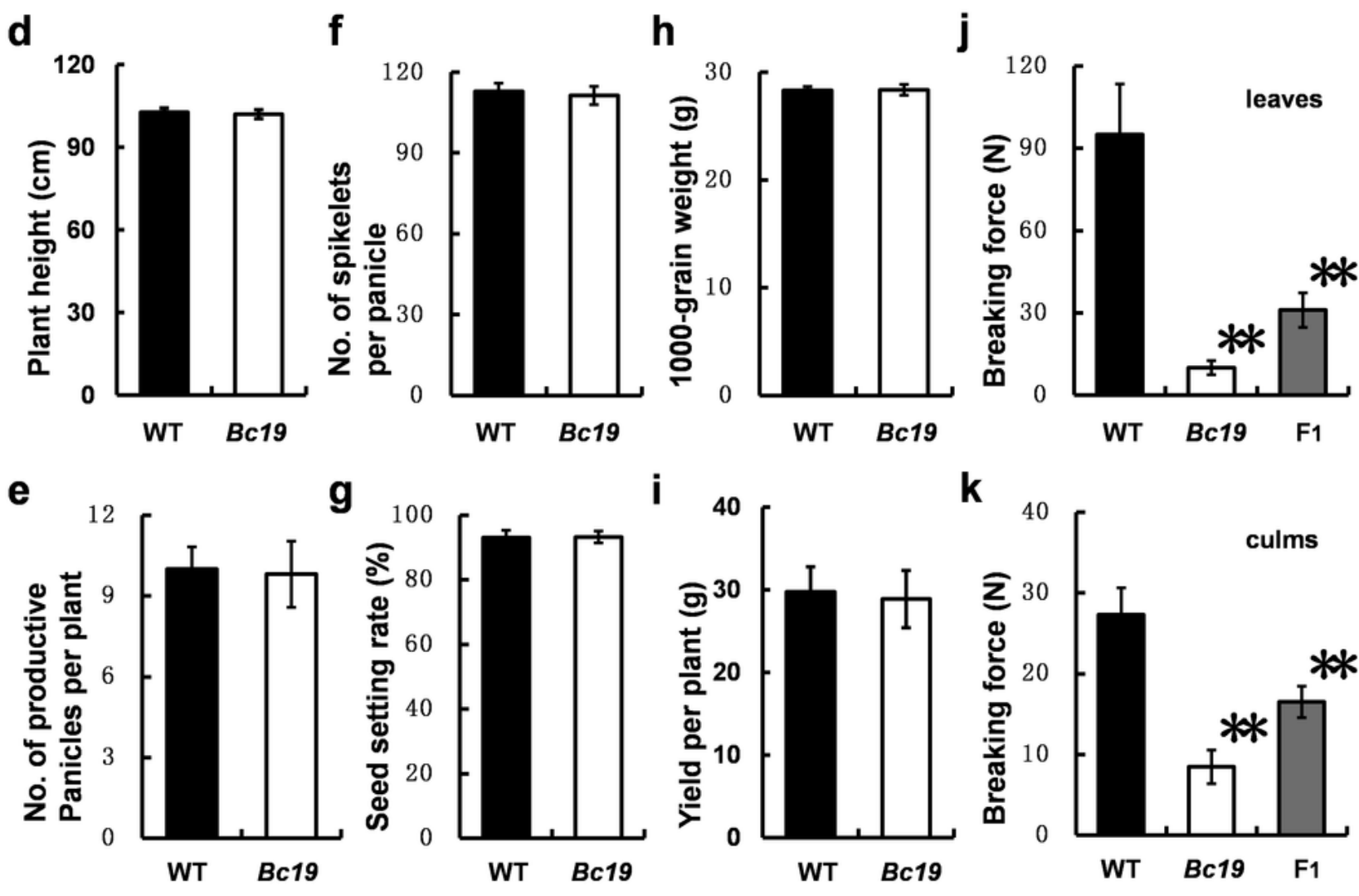

Figure 1

Comparison of phenotypes (a-c), major agronomic traits ( $d-i)$ and breaking force ( $j$ leaves and $k$ culms). a Leaf. $b$ The second internode of main culm from the top. c Plants two weeks after heading. Bars represent standard deviations of three independent measurements. WT: Nipponbare; F1: Obtained from mutant Bc19 crossing with WT. Double-asterisk signifies statistically significant difference compared to the wild type at $\mathrm{P}<0.01$. 

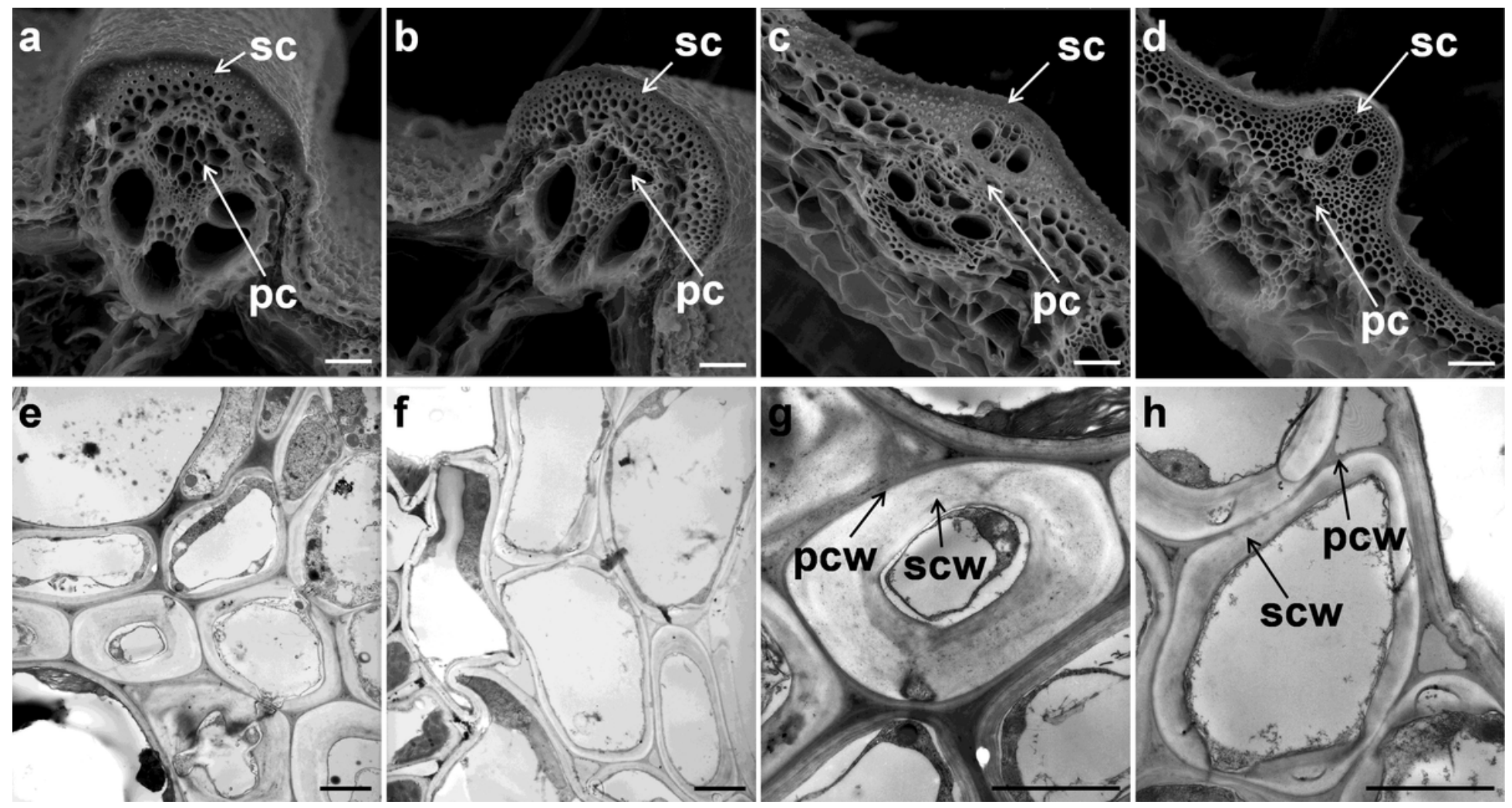

Figure 2

Environmental scanning electron microscope images (a-d) and transmission electron microscope images (e-f) of Bc19 mutant and wild-type plant. a and b Transverse section of the wild-type and Bc19 mutant leaf vein, respectively. $c$ and d Transverse section of the wild-type and Bc19 mutant culm, respectively. $\mathrm{e}$ and $\mathrm{f}$ Sclerenchyma cells of the wild type and Bc19 mutant, respectively. $\mathrm{g}$ and $\mathrm{h}$ Single cell in sclerenchyma tissues of the wild type and Bc19 mutant, respectively. sc, sclerenchyma tissues; pc, parenchymatous tissues; pcw, primary cell wall; scw, secondary cell wall. Scale bar equals $40 \mu \mathrm{m}$ in a-d and $10 \mu \mathrm{m}$ in e-h.
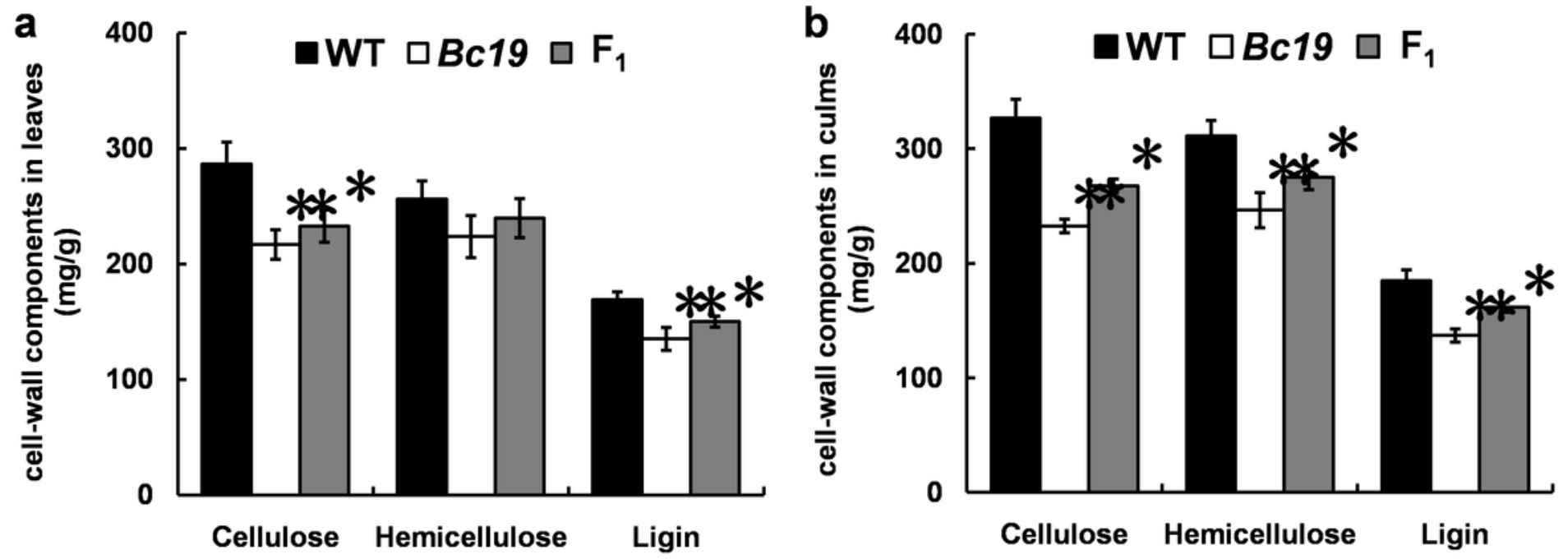

Figure 3 
Contents of cellulose, hemicellulose and lignin in cell walls from leaves (a) and culms (b) of Bc19, the wild type (WT) and F1 plants, in mg g fresh weight-1. F1: Obtained from Bc19 crossing with WT. Double and single Asterisks indicate statistically significant differences compared to the wild type at $P<0.01$ and $P<0.05$, respectively.

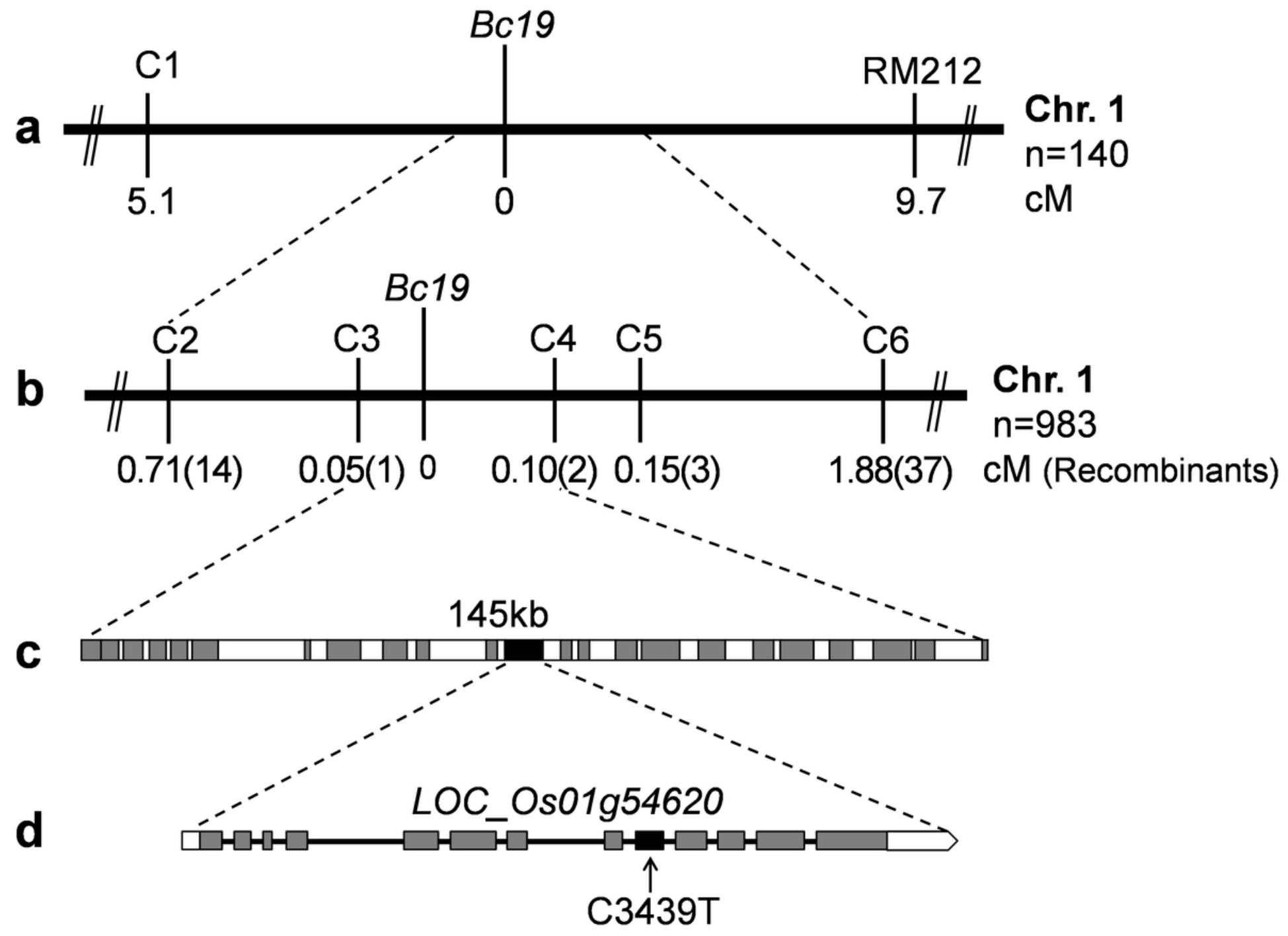

Figure 4

Molecular mapping of Bc19 locus. a The Bc19 locus was mapped between InDel marker C1 and SSR marker RM212 on the long arm of chromosome 1 (Chr.1) of rice using 140 recessive F2 plants. b Bc19 was narrowed between InDel markers C3 and C4 depending on analysis of 983 recessive F2 plants. c 23 annotated genes were found in the 145-kb region between markers C3 and C4. d Structure of the candidate gene Bc19 (LOC_Os01g54620). Gray boxes indicate exons and lines indicate introns. The C3439T point mutation is marked with a black arrow. 


\section{a}

OsCesA4 OsCesA1

OsCesA2

OsCesA3

OsCesA5

OsCesA6

OsCesA7

OsCesA8

OsCesA9

AtCesA1

AtCesA2

AtCesA3

AtCesA4

AtCesA5

AtCesA6

AtCesA7

AtCesA8

AtCesA9

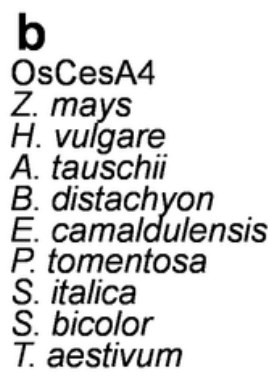

\section{1}

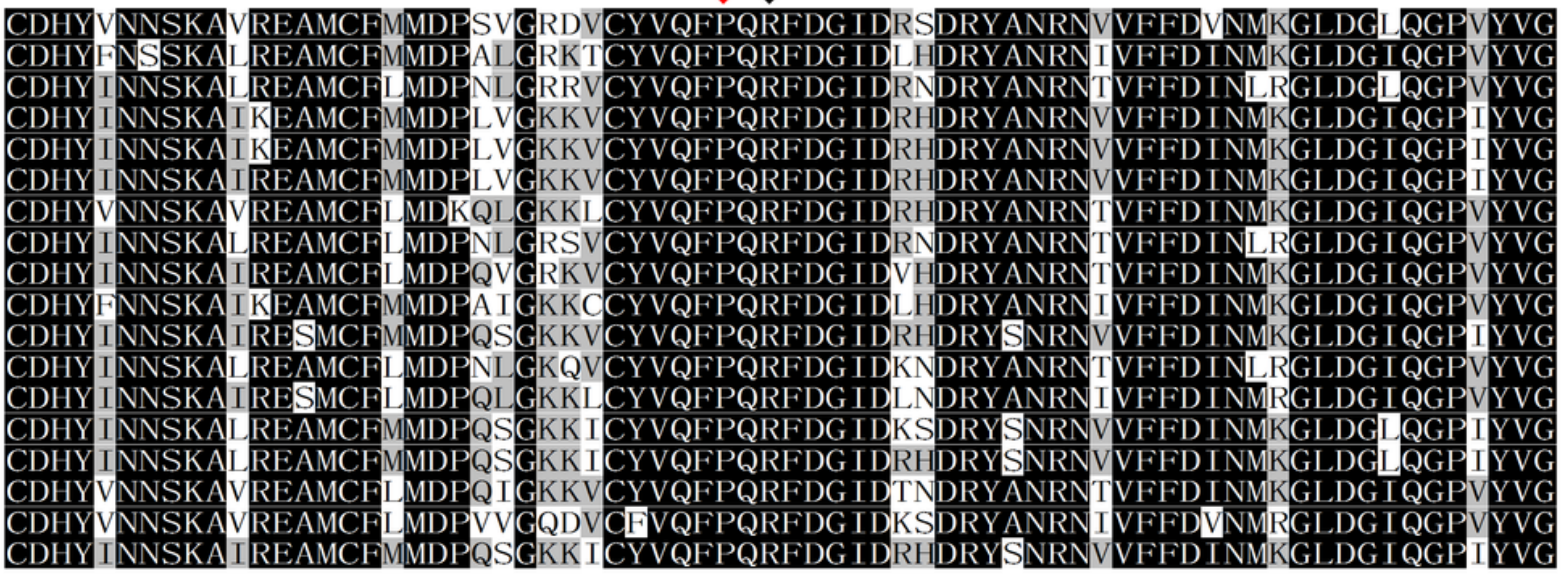

\section{1}

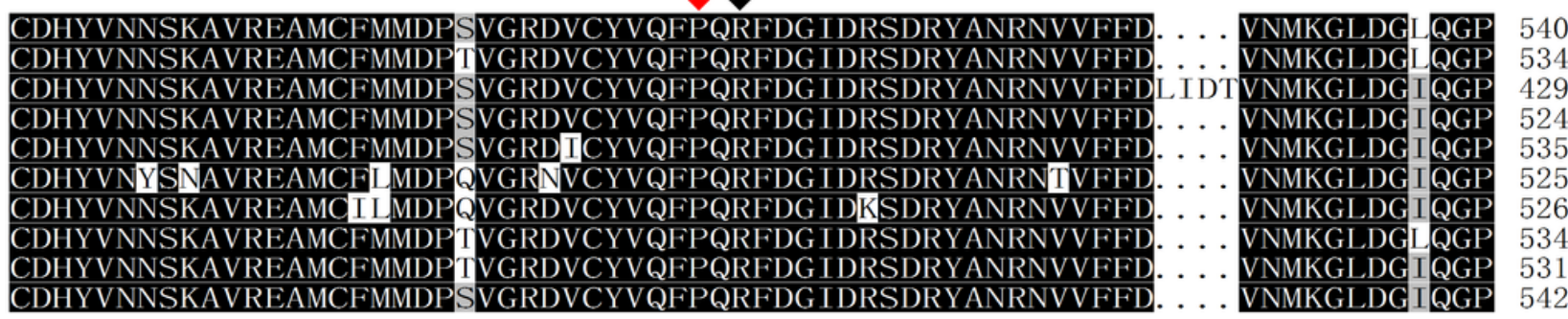

\section{Figure 5}

Part of alignments of amino acid sequences of OsCesA4 with similar sequences in other CesA family members of rice and Arabidopsis (a) and in different species (b). Dark and gray backgrounds showed the identical and similar residues, respectively. A red arrow indicates the missense mutation P507S in mutant $\mathrm{Bc} 19$, which is strictly conserved and is only one residue away from the R588G mutation in mutant Bc6 (black arrow, Kotake et al. 2011). Accession numbers for these sequences are as follows: Oryza sativa OsCesA4 (LOC_0s01g54620, Q5JN63.1), OsCesA1 (LOC_Os05g08370, Q6AT26.1), OsCesA2 (LOC_Os03g59340, Q84M43.1), OsCesA3 (LOC_Os07g24190, Q69V23.1), OsCesA5 (LOC_Os03g62090, Q851L8.1), OsCesA6 (LOC_Os03g62090, Q6YVM4.1), OsCesA7 (LOC_Os10g32980, Q9AV71.1), OsCesA8 (LOC_Os07g10770, Q84ZN6.1), and OsCesA9 (LOC_Os09g25490, Q69P51.1); Arabidopsis thaliana AtCesA1 (At4g32410, NP_194967.1), AtCesA2 (At4g39350, NP_195645.1), AtCesA3 (At5g05170, NP_196136.1), AtCesA4 (At5g44030, Q84JA6.1), AtCesA5 (At5g09870, Q8L778.2), AtCesA6 (At5g64740, Q94JQ6.2), AtCesA7 (At5g17420, NP_197244.1), AtCesA8 (At4g18780, NP_567564.1), and AtCesA9 (At2g21770, Q9SJ22.1); Zea mays (DAA36974.1); Hordeum vulgare (AAR29965.1); Aegilops tauschii (EMT23140.1); Brachypodium distachyon (XP_003569818.1); Eucalyptus camaldulensis (AEK31215.1); Populus tomentosa (AEE60894.1); Setaria italica (XP_004969957.1); Sorghum bicolor (XP_002456361.1); and Triticum aestivum (CBH32503.1). 

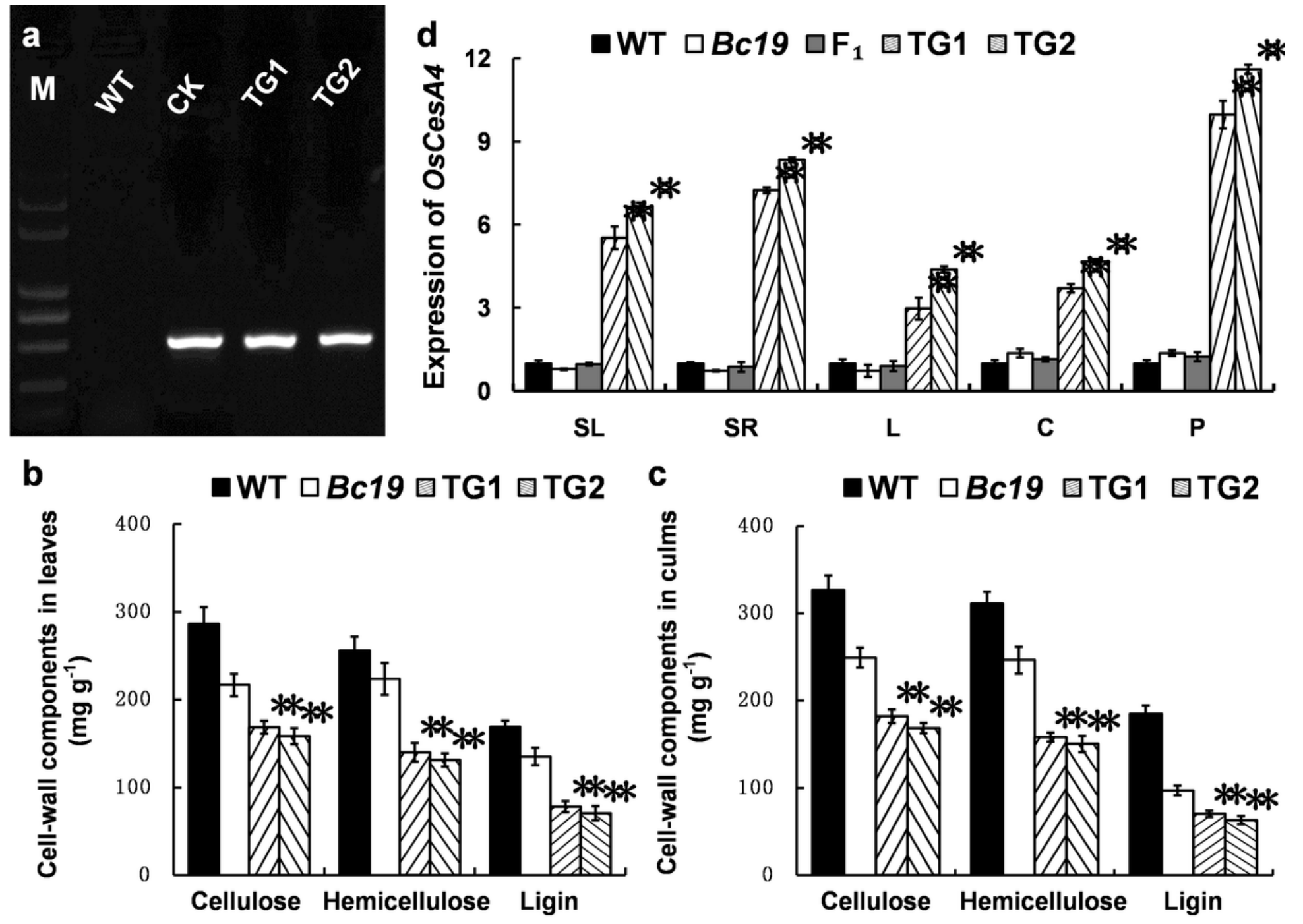

Figure 6

Functional identification of Bc19. a PCR assay of transgenic lines of brittle gene Bc19. M, DL-2000 plus marker; WT, the wild-type parent Nipponbare (the transgenic acceptor, as PCR-negative control); CK, pC2300-Bc19 plasmid (PCR-positive control); TG1 and TG2, positive transgenic lines. b and c Comparison of cell wall components among the wild type plant, mutant Bc19 and two positive transgenic lines, in leaves and culms, respectively. $d$ Transcript levels of OsCesA4 in different tissues of the wild type (WT), the mutant Bc19, the hybrid F1 plants from a cross between WT and Bc19, and two transgenic lines (TG1 and TG2), respectively. SL, seedling leaves; $S R$, seedling roots; $L$ and $C$, leaves and culms from plants two weeks after heading, respectively; P, young spikelets. The expression data of WT were all set to 1.0 and those of other plants were adjusted accordingly. Bars represent standard deviations of three independent experiments. Double asterisks signify statistically significant differences compared to WT at $P<0.01$. 

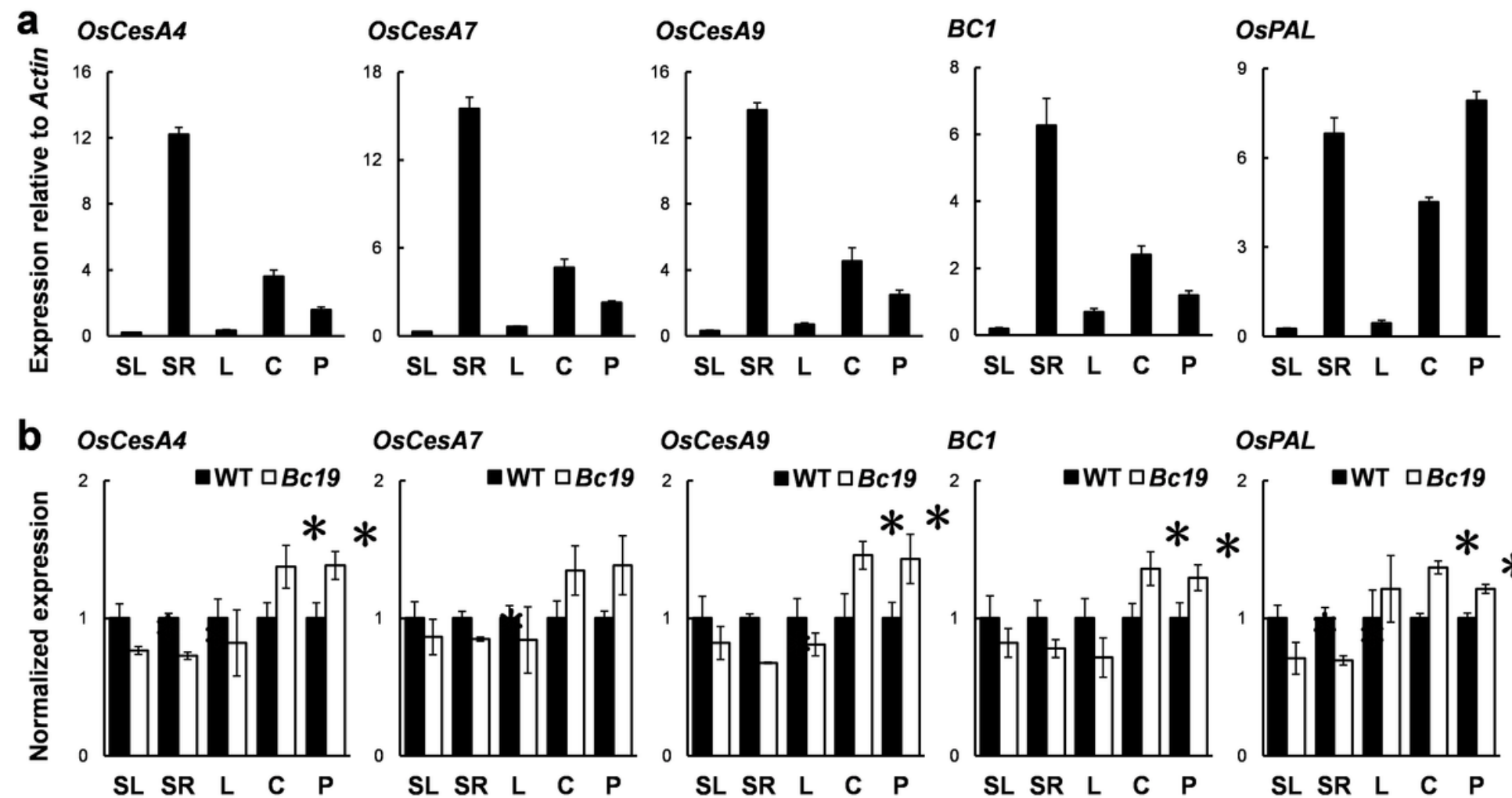

OsCesA7

OsCesA9

$B C 1$

OsPAL
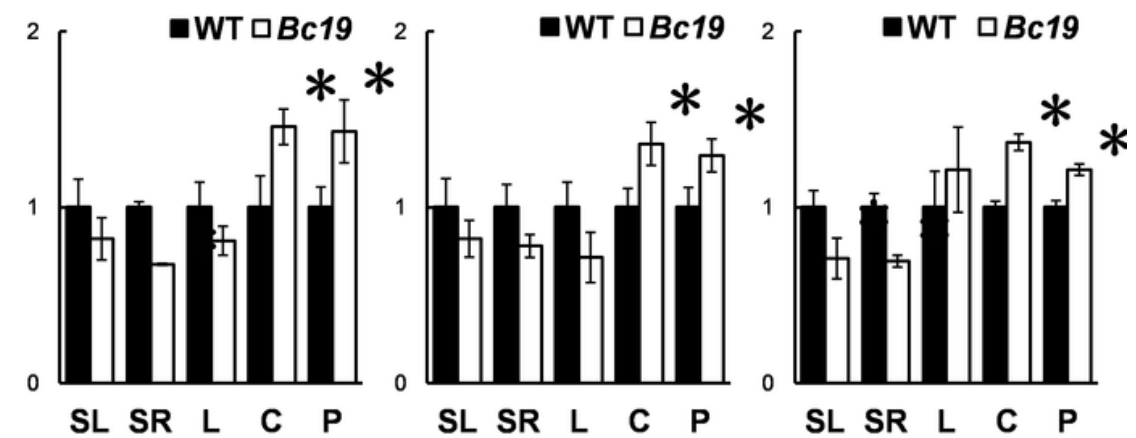

Figure 7

qRT-PCR analysis. Total RNA was isolated from seedling leaves (SL), seedling roots (SR), leaves and culms from plants two weeks after heading ( $L$ and $C)$, young panicles $(P)$. The rice Actin 1 was used as a control. Values represent averages of three independent replicates. Vertical bars show standard errors. a Expression of OsCesAs, BC1 and OsPAL relative to Actin1 in different tissues of the wild type plant. $b$ Comparison of OsCesAs, BC1 and OsPAL mRNAs between WT and Bc19. The expression data of WT were all set to 1.0 and those of Bc19 were adjusted accordingly. Bars show standard deviations. Asterisks signify statistically significant differences compared to WT at $\mathrm{P}<0.05$. 


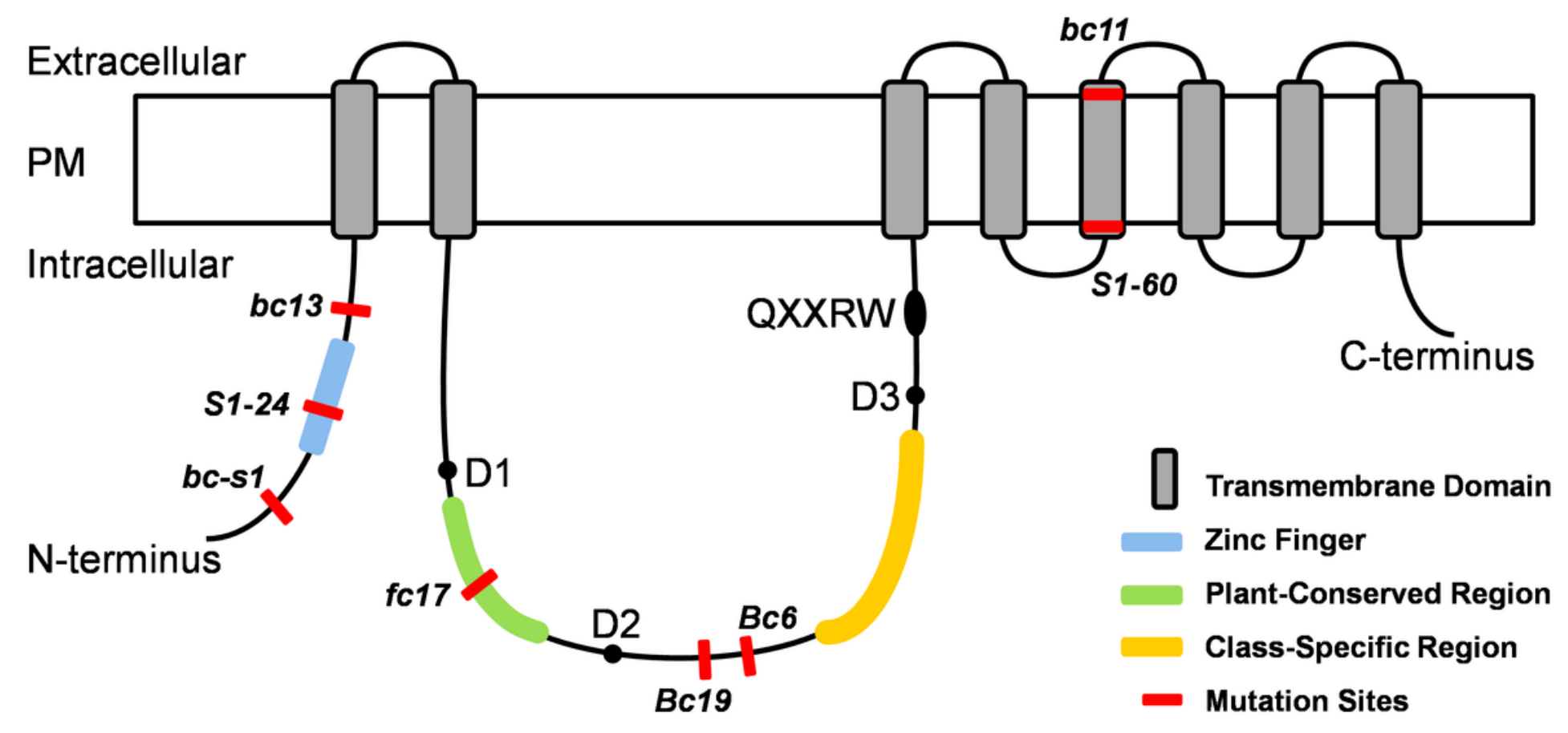

Figure 8

The predicted structure of a CesA protein showing the relative locations of reported point mutations of CesAs in rice. The basic domain structure of CesA was adapted from a review on cellulose synthesis in Arabidopsis (Li et al. 2014b). The open box stands for the plasma membrane (PM). A Zinc-finger domain (a blue bar) exists towards the $\mathrm{N}$-terminus in the first cytoplasmic domain, followed by two transmembrane domains (TMDs; gray bars), the second cytoplasmic domain between the second and the third TMDs, and the other six TMDs. The Plant-conserved Region (P-CR, a green bar), the conserved $D, D, D, Q X X R W$ residues (black spots), and the Class-specific region (C-SR, a yellow bar) are all located in the second cytoplasmic domain. Red bars indicate point missense mutations as follows: bc-s1 (OsCesA9, G10R, Jin et al. 2016), S1-24 (OsCesA7, C40Y, Wang et al. 2016a), bc13 (OsCesA9, G101K, Song et al. 2013), fc17 (OsCesA4, F426S, Li et al. 2018), Bc19 (OsCesA4, P507S) in this study, Bc6 (OsCesA9, R588G, Kotake et al. 2011), S1-60 (OsCesA9, G905D, Wang et al. 2012), and bc11 (OsCesA4, G858R, Zhang et al. 2009).

\section{Supplementary Files}

This is a list of supplementary files associated with this preprint. Click to download.

- Bc19Additionalfile120210704.doc

- Bc19Additionalfile220210707.doc 